\title{
Single-mode versus multimode interferometry: A performance study ${ }^{\star}$
}

\author{
E. Tatulli, P. Mège, and A. Chelli
}

Laboratoire d'Astrophysique, Observatoire de Grenoble, 38041 Grenoble Cedex, France

Received 17 July 2003 / Accepted 6 December 2003

\begin{abstract}
We compare performances of ground-based single-mode and multimode (speckle) interferometers in the presence of partial Adaptive Optics correction of atmospheric turbulence. It is first shown that for compact sources (i.e. sources smaller than the Airy disk of a single telescope) not entirely resolved by the interferometer, the remarkable property of spatial filtering of single-mode waveguides coupled with AO correction significantly reduces the speckle noise which arises from residual wavefront corrugations. Focusing on those sources, and in the light of the AMBER experiment (the near infrared instrument of the VLTI), we show that single-mode interferometry produces a better Signal-to-Noise Ratio on the visibility than speckle interferometry. This is true for bright sources $(K<5)$, and in any case as soon as Strehl ratio of 0.2 is achieved. Finally, the fiber estimator is much more robust - by two orders of magnitude - than the speckle estimator with respect to Strehl ratio variations during the calibration procedure. The present analysis theoretically explains why interferometry with fibers can produce visibility measurements with a very high precision, $1 \%$ or less.
\end{abstract}

Key words. techniques: interferometric - techniques: adaptive optics - methods: analytical

\section{Introduction}

The great interest of using spatial filtering properties of optical waveguides in astronomical interferometers has been proven in the past years (Coudé du Foresto et al. 1997; Berger et al. 2001). As a consequence, integrated optics and fibers are more and more introduced in the design of present and future interferometers to carry the signal to the detector. Furthermore, practical and theoretical studies (Haguenauer 2001; Guyon 2002; Mège et al. 2003) have been undertaken to investigate the physical and optical properties in waveguided interferometers.

The present work aims at comparing the sensitivity and robustness of single-mode and multimode (speckle) interferometry. In Sect. 2, we recall the basic concepts of the fibered interferometric equation and the modal visibility. We derive, in Sect. 3, the formal expression of the Signal to Noise Ratio $(S N R)$ of the modal visibility which takes into account photon, detector and atmospheric noise. In Sect. 4, we propose an analytical approach to estimate the profile of the visibility $S N R$ as a function of the magnitude, from partially Adaptive Optics (AO) corrected interferograms. We also derive the performance of single-mode interferometry applied to the AMBER experiment (the near infrared instrument of the VLTI), in the case of single Gaussian sources. Finally,

Send offprint requests to: E. Tatulli, e-mail: Eric.Tatulli@obs.ujf-grenoble. fr

* Appendices are only available in electronic form at http://wWw.edpsciences.org in Sect. 5, we compute the performances of the multispeckle method (Roddier \& Lena 1984; Mourard et al. 1994) currently used to estimate visibility from non-fibered interferometers and we compare the performances and the robustness of singlemode and speckle interferometry.

\section{The modal visibility}

Mège (2002) theoretically described how the light is carried and processed through an interferometer with optical waveguides. Specifically, he highlighted the coupling phenomenon between the incoming wavefront and the fiber, and analyzed the characteristics of the interferogram recorded on the detector. The principal results concerning the interferometric equation of a $\mathrm{N}$ telescopes $\left(N_{\text {tel }}\right)$ fibered interferometer and the resulting modal visibility can be summarized as following:

$$
\begin{aligned}
I(f)= & \sum_{i}^{N_{\text {tel }}} K_{i} \rho_{i}\left(V_{\star}\right) H_{i}(f) \\
& +\sum_{i}^{N_{\text {tel }}} \sum_{j}^{N_{\text {tel }}} \sqrt{K_{i} K_{j}} \rho_{i j}\left(V_{\star}\right) H_{i j}\left(f-f_{i j}\right)
\end{aligned}
$$

where $I(f)$ is the Fourier transform of the interferogram at the spatial frequency $f, V_{\star}$ is the visibility of the source and $K_{i}$ is the number of photoevents from the $i$ th telescope that would be detected in absence of fibers. $H_{i}(f)$ and $H_{i j}\left(f-f_{i j}\right)$ are the Fourier transforms of the so-called normalized carrying waves centered at respectively the frequencies $f=0$ and $f=f_{i j}$ 
(Mège et al. 2001). Their shape, hence the shape of the interferogram, is entirely determined by the geometry of the fibers. $\rho_{i}\left(V_{\star}\right)$ and $\rho_{i j}\left(V_{\star}\right)$ are respectively the low- $(L F)$ and highfrequency $(H F)$ instantaneous coupling coefficients. They give the fraction of flux entering in the fibers respectively for the photometric and the interferometric channels. Their theoretical expressions are:

$$
\begin{aligned}
\rho_{i}\left(V_{\star}\right) & =\rho_{0} \frac{\left(V_{\star} * T^{i}\right)_{f=0}}{\int T_{0}^{i}(f) \mathrm{d} f} \\
\rho_{i j}\left(V_{\star}\right) & =\rho_{0} \frac{\left(V_{\star} * T^{i j}\right)_{f=f_{i j}}}{\sqrt{\int T_{0}^{i}(f) \mathrm{d} f \int T_{0}^{j}(f) \mathrm{d} f}}
\end{aligned}
$$

where $T^{i}$ and $T^{i j}$ are the (partially AO corrected) modal transfer functions resulting respectively from the normalized autocorrelation and cross-correlation of the entrance aberrationcorrupted pupil weighted by the fiber single mode (Roddier 1988; Mège et al. 2001). $T_{0}^{i}$ is the turbulence-free modal transfer function and $\rho_{0}$ is the optimum coupling efficiency fixed by the fiber core design (Shaklan \& Roddier 1988). Equations (2) and (3) generalize the turbulent coupling efficiency to any kind of sources, i.e. not only when it is unresolved by a single telescope (as first noticed by Dyer \& Christensen (1999) in the non-turbulent case). Note that, for a point source, the low frequency coupling coefficient is proportional to the Strehl ratio $\mathcal{S}, \rho=\rho_{0} \mathcal{S}$ (Coudé du Foresto et al. 2000), and the highfrequency coupling coefficient follows the simple relationship $\left|\rho_{i j}\right|^{2}=\rho_{i} \rho_{j}$.

From Eqs. (2) and (3), we can deduce the expression of the instantaneous modal visibility $V_{i j}^{2}$ at the spatial frequency $f_{i j}$. It is defined as the ratio between the coherent energy (high frequency) and the incoherent one (low frequency):

$V_{i j}^{2}=\frac{\left|\rho_{i j}\left(V_{\star}\right)\right|^{2}}{\rho_{i}\left(V_{\star}\right) \rho_{j}\left(V_{\star}\right)}$.

Perfect equality between the instantaneous modal visibility and the object visibility exists only for point sources $\left(V_{i j}^{2}=V_{*}^{2}=1\right)$. In the general case, however, the instantaneous modal visibility does not match that of the object, especially if the source is extended.

In terms of measurements, the estimator of the modal visibility $\widetilde{V_{i j}^{2}}$ can be computed as the ratio between the interferogram power spectrum at the frequency $f_{i j}:\left|I^{2}\left(f_{i j}\right)\right|=(1-$ $\tau)^{2}\left|\rho_{i j}\left(V_{\star}\right)\right|^{2} K_{i} K_{j}$, and the photometric fluxes: $k_{i}=\tau \rho_{i}\left(V_{\star}\right) K_{i}$, where $\tau$ is the fraction of light selected for photometry at the output of the beam-splitter. Assuming that the latter are estimated independently through dedicated outputs of the optical waveguide (so-called photometric outputs), it holds:

$\widetilde{V_{i j}^{2}}=\frac{\left\langle\left|I^{2}\left(f_{i j}\right)\right|\right\rangle}{\left\langle k_{i} k_{j}\right\rangle}\left(\frac{\tau}{1-\tau}\right)^{2}$.

In the next section, we derive a formal expression of the relative error - the inverse of the $S N R$ - of the modal visibility.

\section{Relative error of the modal visibility}

The noise calculations are based on the spatially continuous model of photodetection introduced by Goodman (1985), (see also Chelli 1989; Berio et al. 2001). Within this framework, the signal is assumed to be corrupted by three different types of noise: (i) the photon noise, with $(1-\tau) K$ and $\tau K$ the total number of detected photoevents in the interferometric and the photometric channels respectively; (ii) the additive Gaussian noise of global variance $\sigma^{2}$ which arises from the detector and from thermal emission; (iii) the atmospheric noise which results from the coupling efficiency variations due to the turbulence.

The noise contributions are derived in Appendices A and B. The square of the relative error of the modal visibility can be described as the sum of three terms:

$\frac{\sigma^{2}\left\{V_{i j}^{2}\right\}}{{\overline{V_{i j}^{2}}}^{2}}=\mathcal{E}_{\mathrm{P}}^{2}(K, \rho)+\mathcal{E}_{\mathrm{A}}^{2}\left(K, \sigma^{2}, \rho\right)+\mathcal{E}_{\mathrm{S}}^{2}(\rho)$,

where $\mathcal{E}_{\mathrm{P}}(K, \rho), \mathcal{E}_{\mathrm{A}}\left(K, \sigma^{2}, \rho\right)$ and $\mathcal{E}_{\mathrm{S}}(\rho)$, are the relative errors due to photon, additive and atmospheric noise, respectively. It holds:

$$
\begin{aligned}
& \mathcal{E}_{\mathrm{P}}^{2}=\left[\frac{N_{\mathrm{tel}}\left(4 \overline{\rho_{l f}\left|\rho_{i j}\right|^{2}}-2 \overline{\rho\left|\rho_{i j}\right|^{2}}\right)}{(1-\tau){\overline{\left|\rho_{i j}\right|^{2}}}^{2}}+\frac{2}{\tau} \frac{\overline{\rho^{2}}}{\bar{\rho}^{3}}\right] \frac{N_{\mathrm{tel}}}{\bar{K}} \\
& +\left[\frac{N_{\mathrm{tel}}^{2}\left(2 \overline{\rho_{l f}^{2}}-\bar{\rho}^{2}\right)}{(1-\tau)^{2}{\overline{\left.\rho_{i j}\right|^{2}}}^{2}}+\frac{4}{(1-\tau)^{2} \overline{\left|\rho_{i j}\right|^{2}}}+\frac{1}{\tau^{2} \bar{\rho}^{2}}\right] \frac{N_{\mathrm{tel}}^{2}}{\bar{K}^{2}} \\
& +\frac{\bar{\rho}}{(1-\tau)^{3} \overline{\left|\rho_{i j}\right|^{2}}} \frac{N_{\text {tel }}^{4}}{\bar{K}^{3}} \\
& \mathcal{E}_{\mathrm{A}}^{2}=\frac{3 N_{\text {pix }} \sigma^{4}+N_{\text {pix }}^{2} \sigma^{4}}{(1-\tau)^{4}{\overline{\left|\rho_{i j}\right|^{2}}}^{2}} \frac{N_{\text {tel }}^{4}}{\bar{K}^{4}}+\frac{2 N_{\text {pix }} \sigma^{2}}{(1-\tau)^{2} \overline{\left|\rho_{i j}\right|^{2}}} \frac{N_{\text {tel }}^{2}}{\bar{K}^{2}} \\
& +\frac{2 N \sigma^{2} \bar{\rho}}{(1-\tau)^{3} \overline{\left|\rho_{i j}\right|^{2}}} \frac{N_{\mathrm{tel}}^{4}}{\bar{K}^{3}} \\
& \mathcal{E}_{\mathrm{S}}^{2}=\frac{\sigma_{\left|\rho_{i j}\right|^{2}}^{2}}{{\overline{\left|\rho_{i j}\right|^{2}}}^{2}}+\frac{\sigma_{\rho_{i} \rho_{j}}^{2}}{\bar{\rho}_{i} \bar{\rho}_{j}}-2 \frac{\operatorname{Cov}\left\{\left|\rho_{i j}\right|^{2}, \rho_{i} \rho_{j}\right\}}{\overline{\left|\rho_{i j}\right|^{2}} \bar{\rho}_{i} \bar{\rho}_{j}}
\end{aligned}
$$

where $\bar{X}$ denotes the expected value of the random quantity $X$, and $\operatorname{Cov}\{X, Y\}$ the covariance between $X$ and $Y . \rho_{l f}$ is the average over all the telescopes of the $L F$ coupling coefficient, and $N_{\text {pix }}$ is the number of pixels per interferogram (see Appendix B for more details).

For bright sources, with the exception of point sources for which $\left|\rho_{i j}\right|^{2}=\rho_{i} \rho_{j}$ (and hence $\mathcal{E}_{\mathrm{S}}(\rho)=0$ ), the dominant noise is the atmospheric noise, which results from the classical speckle noise filtered by the fiber. From now on, we refer to that noise as the modal speckle noise, and the corresponding $S N R,\left(\mathcal{E}_{\mathrm{S}}(\rho)^{-1}\right)$, as the modal speckle $S N R$. This depends on the variation of the $L F$ and $H F$ coupling coefficients and hence, on the strength of the turbulence. As in the speckle case, it does not depend on the source brightness and corresponds to 


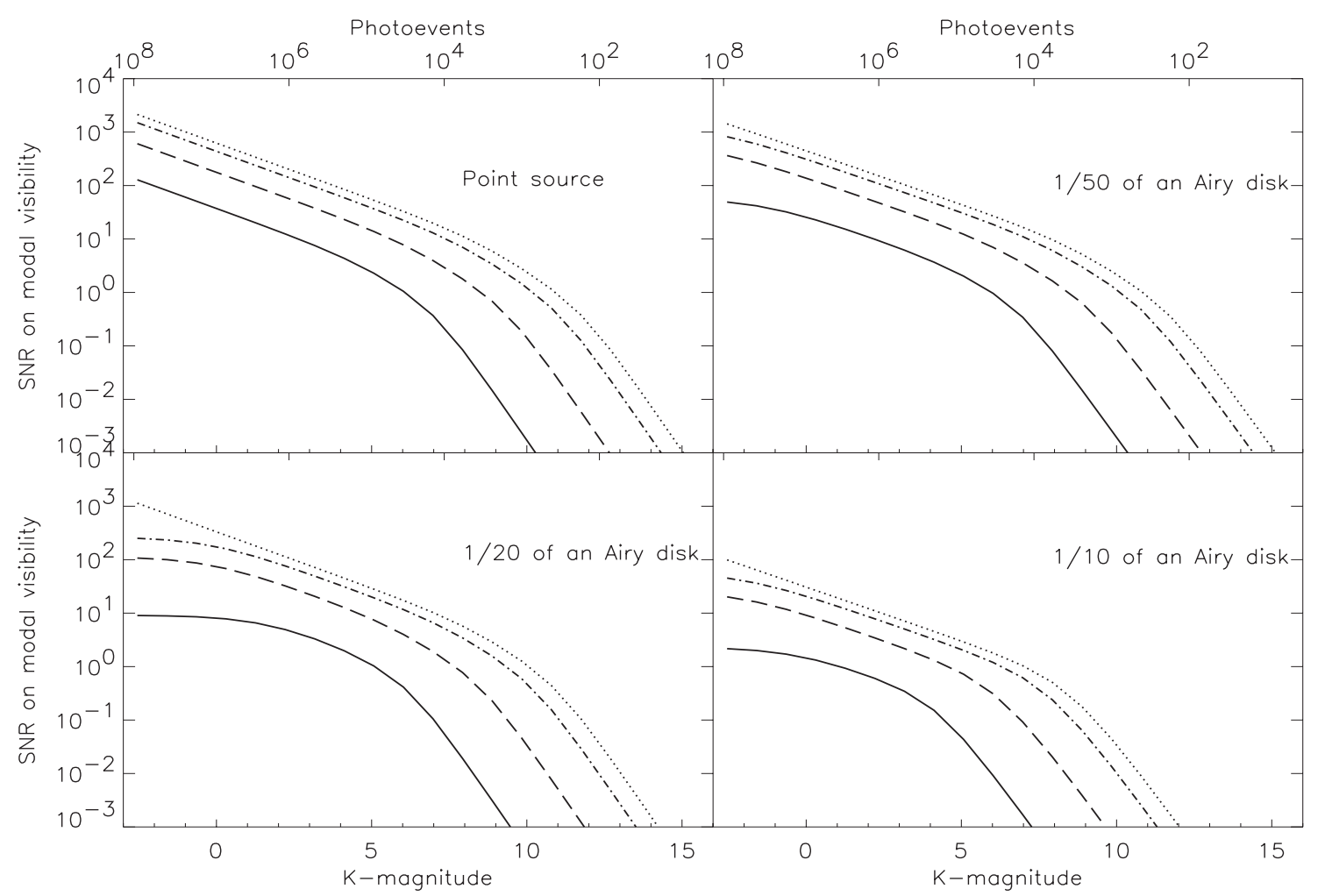

Fig. 1. Modal $S N R$ per interferogram as a function of the magnitude of the source in the $K$ band. From left to right, top to bottom, the object size increases: point source, $1 / 50,1 / 20,1 / 10$ of Airy disk. The considered baseline is $B x=B y=100 \mathrm{~m}$ which mimics an average baseline of the VLTI array. This corresponds to visibility of respectively 1, 0.882, 0.457, 0.04. Results are given for different Strehl ratios: $\mathcal{S}=0.011$ (solid line, no correction), $\mathcal{S}=0.1$ (dashed line), $\mathcal{S}=0.5$ (dash-dotted line) and $\mathcal{S}=0.9$ (dotted line).

the maximum achievable $S N R$ per interferogram. Nevertheless, as we will show, it depends on the source size.

\section{Performances of single-mode interferometry}

In this section, we develop a simple model to estimate the modal speckle noise from partially $\mathrm{AO}$ corrected interferograms, and we derive the performances of single-mode interferometry applied to AMBER, the near-infrared instrument of the Very Large Telescope Interferometer (VLTI).

\subsection{The modal speckle noise}

The derivation of the modal speckle noise is fully detailed in Appendix C: here we recall the outlines of our approach. We assume that: (i) the distance between the telescopes (baseline) is larger than the outer scale of the turbulence, i.e. that the complex amplitudes over pupils $i$ and $j$ are uncorrelated; (ii) the atmospheric phase has Gaussian statistics; (iii) the associated structure function is (spatially) stationary.

At this stage, the modal speckle $S N R$ is described by integrals of dimensions up to sixteen (see Table C.2, from Eqs. (C.11)-(C.15)), which depend on the pupil, the object visibility and the structure functions, respectively. Here we estimate these integrals with a simplified analytical approach dealing with Gaussian functions only. We assume that the object brightness distribution $O_{*}(\alpha)$ and the pupil function have respectively the form:

$O_{*}(\alpha)=\exp \left(-\frac{\alpha^{2}}{\sigma_{\mathrm{o}}^{2}}\right)$

and,

$P(f)=\exp \left(-\frac{f^{2}}{\sigma_{\mathrm{P}}^{2}}\right), \quad \sigma_{\mathrm{P}}=\frac{1}{\sqrt{2}} \frac{D}{\lambda}$

where $D$ is the diameter of the telescope and $\sigma_{\mathrm{p}}$ is defined in such a way that the integral of the Gaussian pupil, i.e. its surface, is equal to the integral of a circular pupil of diameter $D$. Furthermore, in order to take into account the partial correction by adaptive optics, we approximate the structure function $\mathcal{D}(f)$ by:

$\exp \left[-\frac{1}{2} \mathcal{D}(f)\right]=h+(1-h) \mathcal{B}_{\Phi}(f)$

where $h \in[0,1]$ defines the level of correction and $\mathcal{B}_{\Phi}(f)$ is the transfer function of the turbulent atmosphere (Conan 1994) that we will assume Gaussian too:

$\mathcal{B}_{\Phi}=\exp \left(-\frac{f^{2}}{\sigma_{\mathcal{B}}^{2}}\right), \quad \sigma_{\mathcal{B}}=\sqrt{\frac{2}{6.88}} \frac{r_{0}}{\lambda}$

$r_{0}$ being the Fried parameter. Note that, from the previous equations, the Strehl ratio $\mathcal{S}$ is given by:

$\mathcal{S}=h+(1-h) \frac{\sigma_{\mathcal{B}}^{2}}{2 \sigma_{\mathrm{P}}^{2}+\sigma_{\mathcal{B}}^{2}}$ 
At this point, the integrals (C.13)-(C.15) cannot be computed formally yet. To do that, we perform a limited expansion of the expressions under the integrals with respect to the variable $\epsilon=(1-h) / h$. We end up with a series development in $\epsilon^{n}$ in which the coefficients are integrals of Gaussian functions products only. These coefficients are then computed with the MAPLE software. However, the series development converges only for $\epsilon \in[0,1[$ or $h \in] 0.5,1]$, i.e. for good to perfect AO corrections (see Eq. (14)). In order to estimate the modal speckle $S N R$ for average to low AO correction, we compute it in the pure turbulent case $(h=0)$ and we extrapolate from $h=0$ to $h=0.5$. The case $h=0$ is computed separately from standard hypothesis assuming that the complex amplitude of the pure turbulent wavefront follows circular Gaussian statistics (Roddier 1988). We finally obtain an expression of the modal speckle $S N R$ as a function of the major parameters of the observation: the source size, the baseline, the turbulence strength $D / r_{0}$, and the level of $\mathrm{AO}$ correction, i.e. the Strehl ratio $\mathcal{S}$. Strictly speaking, the structure function is not stationary as the error of the AO corrected wavefront increases from the center to the edge of the telescope pupil. Also, its shape is not exactly described by the simplified Eq. (12). Nevertheless, we expect the modal speckle $S N R$ resulting from our model, dealing with Gaussian functions only, to have the right order of magnitude and the correct functional dependencies.

\subsection{Performances}

In this section we compute the $S N R$ profiles of the modal visibility (per interferogram) for faint to bright compact sources (i.e. smaller than the Airy disk of a single telescope). We consider observations under average seeing conditions $\left(r_{0}=\right.$ $1.6 \mathrm{~m})$ with 2 Unit Telescopes $(D=8 \mathrm{~m})$ at the baselines $B x=B y=100 \mathrm{~m}$ and the AMBER recombiner in the $K$ band $(2.2 \mu \mathrm{m})$. We assume that the interferogram is dispersed along the columns of a bi-dimensional detector and that each spectral channel is sampled with 6 pixels to ensure low and high frequency peaks separation. For quantitative calculations, we choose the specific instrumental parameters of AMBER (Malbet et al. 2000; Petrov et al. 2000) together with a spectral resolution of 35 , an integration time of $30 \mathrm{~ms}$ per interferogram, a detector read-out noise of $15 \mathrm{e}^{-} /$pixel and a transmission coefficient $\tau=0.5$. Note that, in those conditions, thermal noise is negligible. We also assumed an optimized instrumental coupling efficiency $\rho_{0}=0.8$ (Shaklan \& Roddier 1988). Figure 1 shows for different Strehl ratios, the modal visibility $S N R$ as a function of the magnitude for 4 object sizes, point source, $1 / 50,1 / 20$ and $1 / 10$ of Airy disk, with respective visibilities $1,0.882,0.457$ and 0.04 at the baselines $B x=B y=100 \mathrm{~m}$.

We can clearly see that the saturation regime, where the modal speckle noise dominates, is significant only in the absence of AO correction. As soon as the image is partially AO corrected, even at small Strehl ratios $(\mathcal{S}>0.1)$, the saturation regime is rejected towards negative magnitudes. It is replaced by an extended "photon noise" regime, which depends on the total flux weighted by the statistics of the coupling

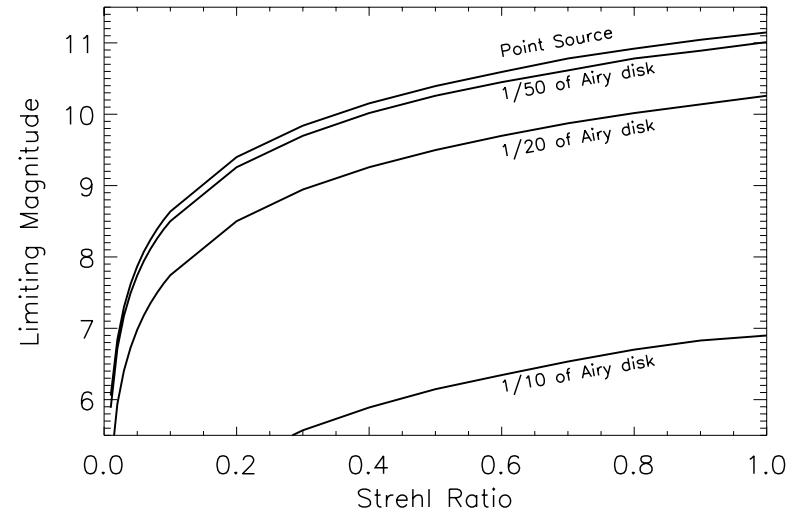

Fig. 2. Instantaneous limiting magnitude of single-mode interferometry technique, defined such that the modal $S N R$ per interferogram is equal to unity. From top to bottom we plot limiting magnitudes for object sizes of respectively: point source, $1 / 50,1 / 20$, and 1/10 of Airy disk. Note that Eq. (15) reproduces fairly well the curves for object sizes equal or smaller than $1 / 20$ of Airy disk.

coefficients, that can be interpreted as transmission coefficients. These transmission coefficients decrease with the Strehl ratio and also when the size of the object increases, therefore lowering the modal visibility $S N R$. At low fluxes, the "detector noise" regime, marked by the break in the $S N R$ slope, takes over.

We define the instantaneous limiting magnitude as the magnitude for which the $S N R$ per interferogram is equal to unity. It occurs at very low fluxes, where the additive noise is dominant. From Eq. (8), and after some simplifications which are valid for partially resolved objects (typically $\sigma_{\mathrm{o}} \geq 1 / 20$ of Airy disk at the considered baseline), the corresponding limiting flux is given by:

$K_{\lim }=\frac{\sqrt{2} \sqrt{N_{\text {pix }}} \sigma N_{\text {tel }}}{(1-\tau) V_{i j} \rho_{0} \mathcal{S}}$.

Figure 2 shows the limiting magnitude as a function of the Strehl ratio for four object sizes: point source, $1 / 50,1 / 20$ and $1 / 10$. Without AO correction, the limiting magnitude is small, between 5 and 6 . As soon as the image is AO corrected and the object partially resolved, the limiting magnitude significantly increases, reaching about 10 for a Strehl ratio of 0.5 . However, for largely resolved objects $(\simeq 1 / 10$ of Airy disk), the coupling efficiency becomes so low that performances of fibered interferometers in terms of $S N R$ and limiting magnitude are severely degraded.

Note, however, that calculations (their detailed description is beyond the scope of this paper) show that the saturation regime can span higher ranges of magnitude in cases where the compact Gaussian source is surrounded by an extended diffuse matter such as a disk or a dust shell. Indeed, it can be shown that, depending on the fraction of the flux in the extended structure, the modal speckle $S N R$ can decrease by an order of magnitude, or more. This is due to the strong fluctuations of the $L F$ coupling coefficient of the extended component. Such behavior is specific to single-mode interferometry, and it demonstrates that fibers are more efficient when they deal with compact sources. On the contrary, we emphasize that in 


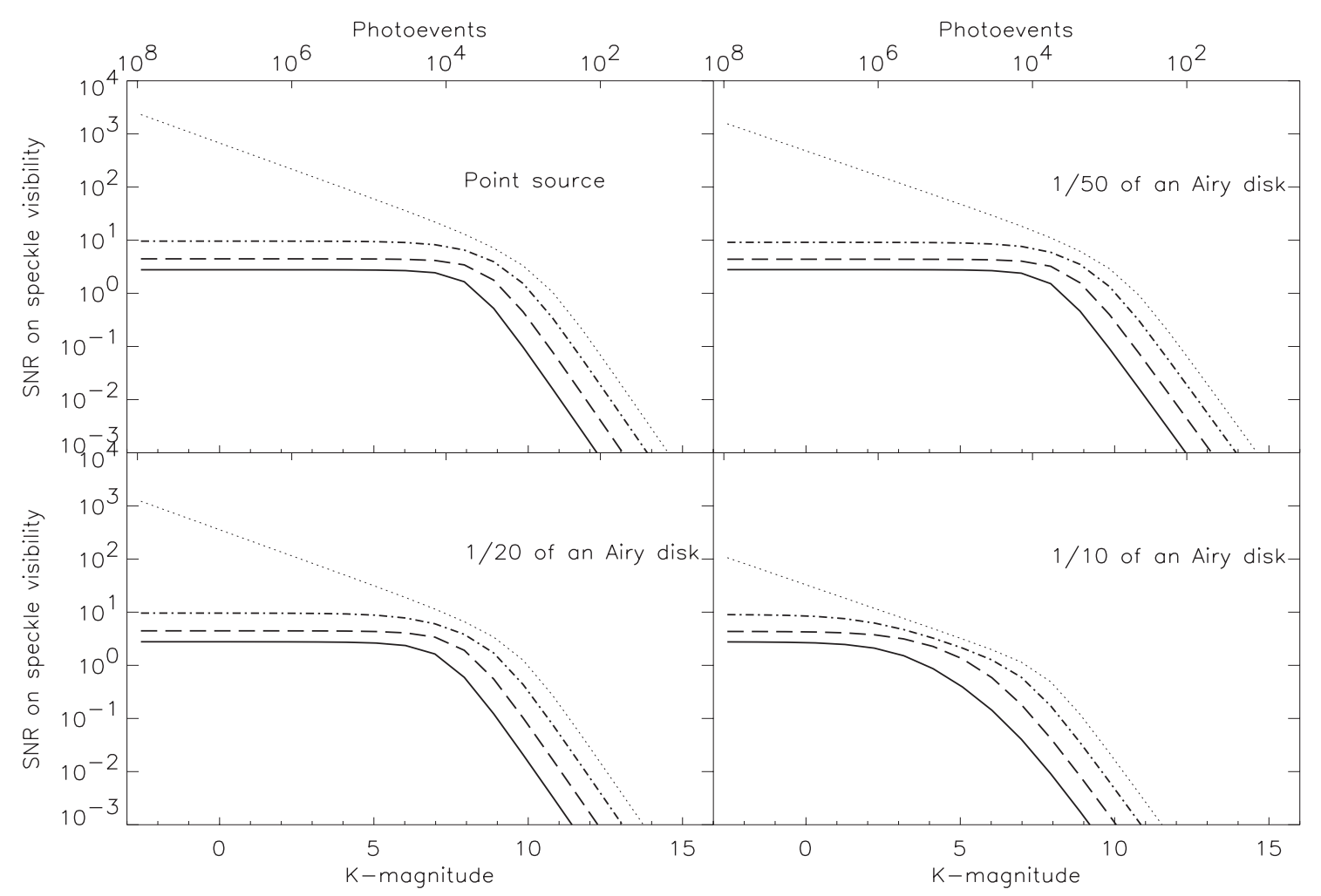

Fig. 3. Multispeckle $S N R$ per interferogram as a function of the magnitude of the source in the $K$ band. As Fig. 1, which those curves must be compared to, the object size is varying from left to right, top to bottom: point source, 1/50,1/20,1/10 of Airy disk, for a baseline $B x=B y=100 \mathrm{~m}$. Results are given for different Strehl ratios: $\mathcal{S}=0.011$ (solid line, no correction), $\mathcal{S}=0.1$ (dashed line), $\mathcal{S}=0.5$ (dash-dotted line) and $\mathcal{S}=0.9$ (dotted line).

classical (multimode) interferometry, a diffuse extended component has no effect on the SNR.

\section{Single-mode versus multimode interferometry}

In this section, we compute the performances of speckle interferometry and we compare their performances and their robustness to those with single-mode interferometry.

\subsection{Performances of speckle interferometry}

In absence of waveguides, visibility estimators can be defined by using speckle techniques, following Labeyrie's method (Labeyrie 1970; Sibille et al. 1979) for single dish observations (see Appendix D). The classical estimator (Roddier \& Lena 1984) consists in taking the ratio of the integral of the high frequency spectral density by the integral of the low frequency one. Taking the integral of the high frequency peak is essential to perform a consistent comparison with the fiber case, since fibered interferometry induces an average (more precisely a convolution) of the visibility over the high frequency (see Eq. (3)). Moreover, to insure a thoroughly consistent comparison, we assume that the photometric fluxes are measured simultaneously with the interferograms (instead of taking the integral of the low frequency spectral density) and we define the estimator of visibility as the ratio between the integrated high frequency peak of the spectral density and the photometric fluxes. It may be written as:

$$
\widetilde{V^{2}}\left(f_{i j}\right) \propto \frac{\int_{f_{i j}-\frac{D}{\lambda}}^{f_{i j}+\frac{D}{\lambda}}|I(f)|^{2} \mathrm{~d} f}{\left\langle k_{i} k_{j}\right\rangle} .
$$

This estimator needs to be calibrated by a point source. It has basically the same performance than the classical speckle estimator, but not the same robustness, as we will see later. In practice, we replace the integral by a discrete sum with a regular spacing $\Delta f=\frac{r_{0}}{\lambda}$. Since we consider partial correction by Adaptive Optics, the points involved in the discrete sum are not statistically independent. Their correlations are taken into account in the signal to noise ratio calculations, detailed in Appendix D. These calculations require the knowledge of the first and second order statistics of the speckle transfer function. Formal expressions of those moments have been derived following a procedure similar to the one described in Sect. 4.1. For numerical applications, we adopted the same parameters than for single-mode interferometry, with the exception of the number of pixels required to correctly sample the interferograms, which is $N=6\left(D / r_{0}\right)$ for 2 telescopes (Chelli \& Mariotti 1986). Figure 3 shows, for different Strehl ratios, the speckle visibility $S N R$ as a function of the magnitude, for the four object sizes previously considered. The striking difference with single-mode interferometry is the almost absence of the "photon noise" regime, even with AO correction. 


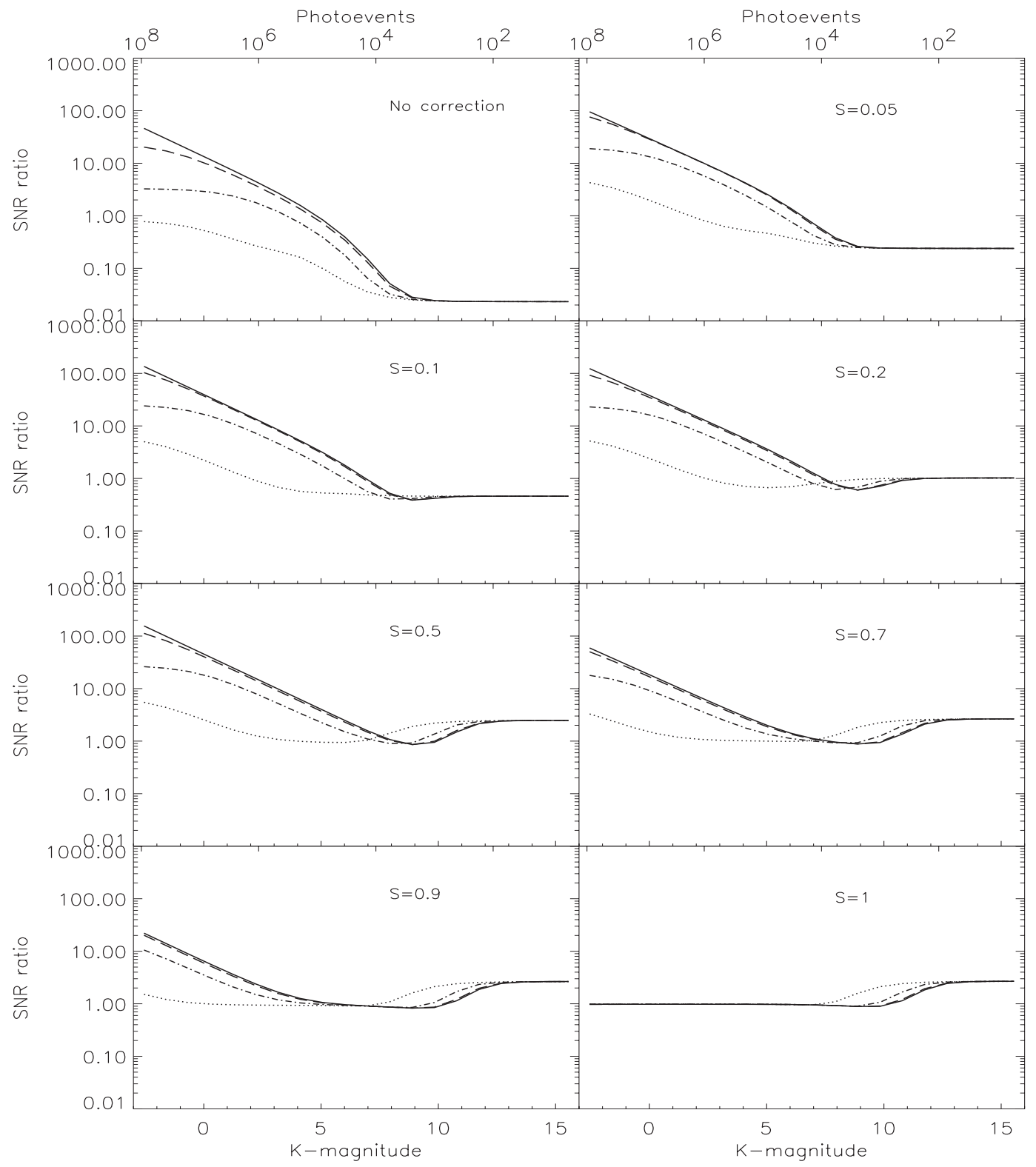

Fig. 4. Ratio between fibered and multispeckle visibility $S N R$ as a function of the magnitude for different AO correction levels, from left to right, top to bottom: $\mathcal{S}=0.011$ (no correction), $\mathcal{S}=0.05, \mathcal{S}=0.1, \mathcal{S}=0.2, \mathcal{S}=0.5, \mathcal{S}=0.7, \mathcal{S}=0.9$ and $\mathcal{S}=1$. Ratios are displayed for different source sizes in fraction of Airy disk: point source (solid line), 1/50 (dashed line), 1/20 (dash-dotted line), 1/10 (dotted line).

Instead, the saturation regime is reached in the entire range of magnitudes until the "detector noise" regime takes over. Note, furthermore, that the speckle noise is barely dependent on the source size and does not cancel out for a point source, contrarily to the modal speckle noise.

\subsection{Comparison of performances and robustness}

Figure 4 shows the ratio between single-mode and speckle $S N R$ as a function of the magnitude, for different compact source sizes and Strehl ratios. Clearly, with the exception of bright sources $(K<5)$, speckle interferometry without AO correction is superior to single-mode interferometry. However, as the degree of $\mathrm{AO}$ correction increases, the situation very rapidly evolves, and, starting from a Strehl ratio of 0.2, single-mode interferometry always reaches higher $S N R$ than speckle interferometry. Such efficiency of fibered interferometry is due to two major aspects: the remarkable property of spatial filtering of the fiber as soon as enough coherent energy (typically 20\%) lies in the Airy disk, together with the possibility of sampling the signal on few pixels. On the contrary, multimode interferometry requires at least good $\mathrm{AO}$ correction $(\mathcal{S}>0.5)$ to significantly reduce the speckle noise. Moreover, fringe sampling is seeing dependent and requires a much larger number of pixels, specially for telescopes with large apertures such as the VLTI. Figure 5 compares the limiting magnitudes of both methods. Without AO correction, the speckle limiting magnitude is between 8 and 9, well above the single-mode one. With 


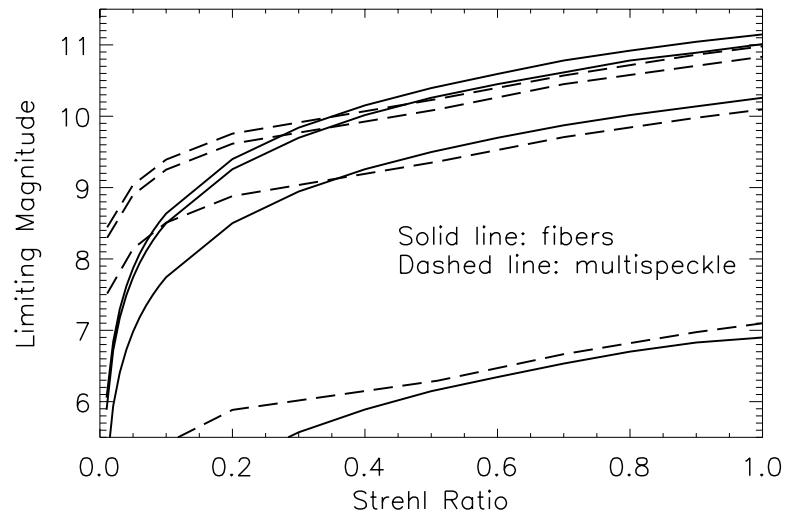

Fig. 5. Comparison of fibered and speckle interferometry limiting magnitudes. Limiting magnitude is still defined such that $S N R=1$. Solid lines and dashed lines show fiber and speckle cases respectively. From top to bottom, the object size is: point source, 1/50, 1/20, and $1 / 10$ of Airy disk.

AO correction and Strehl ratios larger than 0.2, the limiting magnitudes are basically the same.

To evaluate the robustness of each method, we investigate the stability of the measured visibility versus the Strehl ratio. Figure 6 shows the visibility variation as a function of the Strehl ratio, normalized to the visibility at $\mathcal{S}=0.7$. The two upper curves correspond to the speckle estimator studied above and to the classical speckle estimator (ratio of the integrated high and low frequency peaks of the spectral density), respectively. The classical speckle estimator is much more robust, but, in both cases, even a small Strehl ratio variation (0.2) can produce visibility variations up to $10 \%$. The 3 lower curves correspond to the modal visibility estimator for source sizes $1 / 50$, $1 / 20$ and $1 / 10$ of Airy disk (point source is irrelevant since it is theoretically independent of the turbulence). The robustness of the modal estimator depends on the source size, while the multispeckle estimator does not. In any case, however, the modal estimator is clearly more robust than the speckle one, by more than 2 orders of magnitude. The modal visibility is stable at a level less than $1 \%$ over all the range of possible Strehl ratios, from 0 to 1 . This last property is interesting, not only to perform high precision measurements, but also for the selection of reference sources. Indeed, it would suggest that the use of a reference source having a large magnitude difference with the studied object, and hence, where the level of AO correction is different, does not affect the precision of the measurement.

Note, however, that this comparison focuses on compact sources. For a central source surrounded by a larger diffuse component, the fluctuations of the $L F$ coupling coefficient due to the extended structure severely reduces the filtering properties of the single-mode fibers. As a consequence, a situation similar to the multispeckle case occurs with a saturation regime spanning a large range of magnitudes, and where the larger the fraction of flux in the extended component, the larger the range. The presence of an extended structure also causes a decrease of the robustness, which, however, remains better than in the case of speckle estimators, by one order of magnitude.

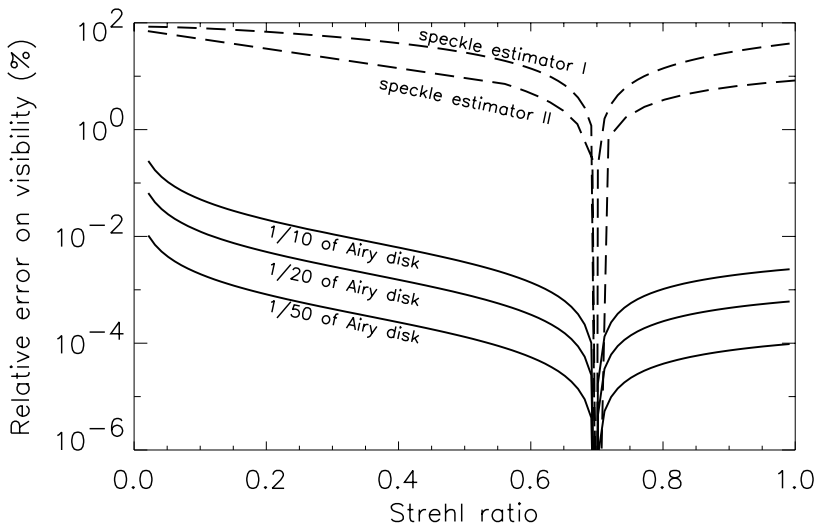

Fig. 6. Robustness of single-mode and multimode estimators as a function of the Strehl ratio. Comparison is given in terms of relative error on the visibility $\Delta V / V$. Solid lines deal with fiber estimator for respectively $1 / 10,1 / 20$ and $1 / 100$ of Airy disk, top to bottom. Dashed lines gives the robustness of two speckle estimators. Top: so-called estimator I which takes into account the estimator defined in this paper (Eq. (16)). Bottom: estimator II describes the "classical" speckle estimator (see text). Note that both speckle estimators are object size independent.

\section{Summary}

In this paper, we have developed a formalism that can predict theoretical $S N R$ on visibilities, when measurements are partially AO corrected and are corrupted by photon, additive (detector, thermal) and residual atmospheric noise. This formalism has been applied to single-mode and multimode (speckle) interferometers. We have assumed that: (i) the wavefronts over two distinct telescopes are uncorrelated; (ii) the atmospheric phase has Gaussian statistics, and the associated structure function is (spatially) stationary; (iii) the pupil function, the object brightness distribution, and the transfer function of the turbulent atmosphere are Gaussian. In the case of single-mode interferometry and for compact sources (i.e. sources smaller than the Airy disk of a single telescope) not entirely resolved by the interferometer, we show that, in the presence of AO correction, the remarkable filtering properties of fibers reject the SNR saturation regime due to speckle noise towards negative magnitudes. Instead, the modal visibility $S N R$ is dominated by the "photon noise" regime followed by a break close to the limiting magnitude due to "detector noise". For AMBER, the near infrared experiment of the VLTI, we found that the limiting magnitude is about $K=10$ for a Strehl ratio of 0.5 , with two $8 \mathrm{~m}$ Unit Telescopes (typical atmospheric and instrumental parameters of the VLTI/AMBER instrument were discussed in Sect. 4.2). In the case of speckle interferometry, the visibility $S N R$ is characterized by the almost absence of the "photon noise" regime, replaced by the saturation regime due to speckle noise. With the exception of bright sources $(K<5)$, without AO corrections speckle interferometry reaches higher SNR than singlemode interferometry, leading to higher limiting magnitudes. However, the situation is different in presence of AO correction, even small $(\mathcal{S}=0.2)$ : the limiting magnitude of both methods becomes the same, but the single-mode reaches much higher SNR than speckle interferometry. This last property, together 
with the insensitivity of the modal visibility to the Strehl ratio variations, explains why interferometry with fibers can produce visibility measurement with a very high precision of $1 \%$ or less, on compact sources.

\section{References}

Berger, J.-P., Haguenauer, P., Kern, P., et al. 2001, A\&A, 376, 31 Berio, P., Mourard, D., Pierron, M., \& Chelli, A. 2001, J. Opt. Soc. Am., 18, 614

Chelli, A., \& Mariotti, J. M. 1986, A\&A, 157, 372

Chelli, A. 1989, A\&A, 225, 277

Conan, J.-M. 1994, Ph.D. Thesis, Univerté Paris XI Orsay

Coudé du Foresto, V., Perrin, G., Mariotti, J.-M., Lacasse, M., \& Traub, W. 1997, in Integrated Optics for Astronomical Interferometry, 115

Coudé du Foresto, V., Faucherre, M., Hubin, N., \& Gitton, P. 2000, A\&AS, 145, 305

Dyer, S. D., \& Christensen, D. A. 1999, J. Opt. Soc. Am. A, 16, 2275

Goodman, J. W. 1985, Statistical Optics (New York: Wiley)

Guyon, O. 2002, A\&A, 387, 366

Haguenauer, P. 2001, Ph.D. Thesis, Université de Grenoble
Korff, D. 1973, J. Opt. Soc. Am., 63, 971

Labeyrie, A. 1970, A\&A, 6, 85

Malbet, F., et al. 2000, AMBER Instrument Analysis Report, Technical Report VLT-TRE-AMB-15830-00000-0001, ESO Preliminary Design Review

Mège, P., Malbet, F., \& Chelli, A. 2001, in SF2A-2001: Semaine de l'Astrophysique Francaise, ed. F. Combes, D. Barret, \& F. Thévenin (EDP Sciences), 581

Mège, P. 2002, Ph.D. Thesis, Université de Grenoble

Mège, P., Malbet, F., \& Chelli, A. 2003, Proc. SPIE, 4838, 329

Mourard, D., Tallon-Bosc, I., Rigal, F., et al. 1994, A\&A, 288, 675

Papoulis, A. 1984, Probability, random variables and stochastic processes (New York: McGraw-Hill), 2nd ed.

Petrov, R. G., Malbet, F., Richichi, A., et al. 2000, Proc. SPIE, 4006, 68

Roddier, F., \& Lena, P. 1984, JOpt, 15, 171

Roddier, F. 1988, in Diffraction-limited Imaging with Very Large Telescopes, ed. D. M. Alloin, \& J.-M. Mariotti (Kluwer Academic Publishers), 1989, 33

Shaklan, S., \& Roddier, F. 1988, ApOpt, 27, 2334

Sibille, F., Chelli, A., \& Lena, P. 1979, A\&A, 79, 315

Tallon, M., \& Tallon-Bosc, I. 1992, A\&A, 253, 641 


\section{Online Material}




\section{Appendix A: Moments of the spectral density of an interferogram}

\section{A.1. Formalism}

In order to compute the moments of the spectral density, we use the spatially continuous model of photodetection process of Goodman (1985) where the detected signal takes the form:

$\mathrm{d}(x, y)=\sum_{n=1}^{K} \delta\left(x-x_{n}, y-y_{n}\right)$

and its Fourier transform:

$\widehat{D}\left(f_{x}, f_{y}\right)=\sum_{n=1}^{K} \mathrm{e}^{-2 i \pi\left(f_{x} x_{n}+f_{y} y_{n}\right)}$

The position $\left(x_{n}, y_{n}\right)$ as well as the number of photoevents $K$ are considered as independent random processes with probability density functions proportional to the intensity $I(x, y)$. This formalism has been deeply studied (Goodman 1985; Chelli 1989) and the results already proven will not be re-demonstrated here. This appendix especially focuses on the original approach where the signal is corrupted by an additive noise $\epsilon$, following a centered Gaussian random process, and of Fourier transform $\widehat{\epsilon}$. Using a mono-dimensional writing, but without loss of generality, the corrupted signal $\widehat{S}(f)$ has now the expression:

$\widehat{S}(f)=\widehat{D}(f)+\widehat{\epsilon}(f)=\sum_{n=1}^{K} \mathrm{e}^{-2 i \pi f x_{n}}+\widehat{\epsilon}(f)$.

We compute the spectral density statistics with respect to the additive Gaussian noise, the photon noise and the turbulent atmosphere, respectively. The estimator of the spectral density is:

$Q(f)=|\widehat{S}(f)|^{2}$.

\section{A.2. Expected value}

We first take the expected value with respect to the additive noise from Eq. (A.3). It becomes:

$\mathrm{E}_{\epsilon}\{Q(f)\}=|\widehat{D}(f)|^{2}+\mathrm{E}_{\epsilon}\left\{\mid \hat{\epsilon}^{2}\right\}=|\widehat{D}(f)|^{2}+N \sigma^{2}$.

Then computing $|\widehat{D}|^{2}$ from Eq. (A.2):

$|\widehat{D}(f)|^{2}=\sum_{n=1}^{K} \sum_{l=1}^{K} \mathrm{e}^{-2 i \pi\left(f\left[x_{n}-x_{l}\right]\right)}$.

Goodman formalism finally leads to a total expected value of:

$\mathrm{E}\{Q(f)\}=\left\langle\left.\bar{K}^{2} \widehat{\mid i}(f)\right|^{2}\right\rangle_{\phi}+\langle\bar{K}\rangle_{\phi}+N \sigma^{2}$

where \langle\rangle$_{\phi}$ denotes the expected value relatively to the turbulent atmosphere random process.

\section{A.3. Variance}

The variance is defined by:

$\sigma^{2}\{Q\}=\mathrm{E}\left\{Q^{2}\right\}-\mathrm{E}\{Q\}^{2}$.

Computing $\mathrm{E}\left\{Q^{2}\right\}$ with respect to the additive noise, we have:

$\mathrm{E}\left\{Q^{2}\right\}=\mathrm{E}\left\{|\widehat{D}|^{4}\right\}+4 N \sigma^{2} \mathrm{E}\left\{|\widehat{D}|^{2}\right\}+2 N^{2} \sigma^{4}$

from which Goodman formalism in the pure photon noise case can be applied. Finally the variance of the spectral density may be written as:

$$
\begin{aligned}
\sigma^{2}\{Q(f)\}= & \left\langle\left.\bar{K}^{4} \widehat{\mid i}(f)\right|^{4}\right\rangle_{\phi}-\left\langle\left.\bar{K}^{2} \widehat{\mid i}(f)\right|^{2}\right\rangle_{\phi}^{2} \\
& +4\left\langle\left.\bar{K}^{2} \widehat{\mid i}(f)\right|^{2}\right\rangle_{\phi}-2\langle\bar{K}\rangle_{\phi}\left\langle\left.\bar{K} \widehat{\mid i}(f)\right|^{2}\right\rangle_{\phi} \\
& +4\left\langle\left.\bar{K}^{2} \widehat{\mid i}(f)\right|^{2}\right\rangle_{\phi}+2\left\langle\bar{K}^{2}\right\rangle_{\phi}-\langle\bar{K}\rangle_{\phi}^{2}+3 N \sigma^{4} \\
& +N^{2} \sigma^{4}+2 N \sigma^{2}\langle\bar{K}\rangle_{\phi}+2 N \sigma^{2}\left\langle\left.\bar{K}^{2} \widehat{\mid i}(f)\right|^{2}\right\rangle_{\phi}
\end{aligned}
$$

\section{A.4. Covariance}

We can also compute the covariance of the spectral density which is defined as:

$\operatorname{Cov}\left\{Q_{1}, Q_{2}\right\}=\mathrm{E}\left\{Q_{1} Q_{2}\right\}-\mathrm{E}\left\{Q_{1}\right\} \mathrm{E}\left\{Q_{2}\right\}$.

It becomes:

$$
\begin{aligned}
\operatorname{Cov}\left\{Q\left(f_{1}\right), Q\left(f_{2}\right)\right\}= & \left\langle\left.\bar{K}\left|\widehat{i}\left(f_{1}\right)\right|^{2} \widehat{i}\left(f_{2}\right)\right|^{2}\right\rangle_{\phi} \\
& -\left\langle\left.\bar{K}^{2} \widehat{\mid i}\left(f_{1}\right)\right|^{2}\right\rangle_{\phi}\left\langle\left.\bar{K}^{2} \widehat{\mid i}\left(f_{2}\right)\right|^{2}\right\rangle_{\phi} \\
& +\left\langle\bar{K}^{3} \widehat{i}\left(f_{1}\right) \hat{i}^{*}\left(f_{2}\right) i^{*}\left(f_{1}-f_{2}\right)\right\rangle_{\phi} \\
& +\left\langle\bar{K}^{3} \widehat{i}\left(f_{1}\right) \widehat{i}\left(f_{2}\right) \widehat{i}\left(f_{1}-f_{2}\right)\right\rangle_{\phi} \\
& +\left\langle\bar{K}^{3} \widehat{i}\left(f_{1}\right) \widehat{i}\left(f_{2}\right) \hat{i}^{*}\left(f_{1}+f_{2}\right)\right\rangle_{\phi} \\
& +\left\langle\bar{K}^{3} \widehat{i}^{*}\left(f_{1}\right) \widehat{i}^{*}\left(f_{2}\right) \widehat{i}\left(f_{1}+f_{2}\right)\right\rangle_{\phi} \\
& +\left\langle\left.\bar{K}^{3} \widehat{\mid i}\left(f_{1}\right)\right|^{2}\right\rangle_{\phi}-\langle\bar{K}\rangle_{\phi}\left\langle\left.\bar{K}^{2} \widehat{\mid i}\left(f_{1}\right)\right|^{2}\right\rangle_{\phi} \\
& +\left\langle\left.\bar{K}^{3} \widehat{\mid i}\left(f_{2}\right)\right|^{2}\right\rangle_{\phi}-\langle\bar{K}\rangle_{\phi}\left\langle\left.\bar{K}^{2} \widehat{\mid i}\left(f_{2}\right)\right|^{2}\right\rangle_{\phi} \\
& +2\left\langle\left.\bar{K}^{2} \widehat{\mid i}\left(f_{1}\right)\right|^{2}\right\rangle_{\phi}+2\left\langle\left.\bar{K}^{2} \widehat{\mid i}\left(f_{2}\right)\right|^{2}\right\rangle_{\phi} \\
& +\left\langle\left.\bar{K}^{2} \widehat{\mid i}\left(f_{1}-f_{2}\right)\right|^{2}\right\rangle_{\phi}+\left\langle\left.\bar{K}^{2} \widehat{\mid i}\left(f_{1}+f_{2}\right)\right|^{2}\right\rangle_{\phi} \\
& +\left\langle\bar{K}^{2}\right\rangle-\langle\bar{K}\rangle_{\phi}^{2}+\langle\bar{K}\rangle_{\phi}+3 N \sigma^{4} .
\end{aligned}
$$


E. Tatulli et al.: Single-mode interferometry, Online Material p 3

\section{Appendix B: Estimator of the visibility for fibered interferometers}

\section{B.1. The interferometric equation}

Mège (2002) has shown that the fibered interferometric equation could be written as follows:

$I(f)=\sum_{i} K_{i} \rho_{i} H_{i}(f)+\sum_{i} \sum_{j} \sqrt{K_{i} K_{j}} \rho_{i j} H_{i j}\left(f-f_{i j}\right)$

where $\rho_{i}$ and $\rho_{i j}$ are respectively the low and high frequency coupling efficiencies and $K_{i}$ is the number of detected photoevents in the absence of a fiber on the $i$ th telescope. Moreover we define $k_{i}$ as the number of photoevents at the output of the fiber. We have the relationship $k_{i}=\rho_{i} K_{i}$. For simplicity purposes, we also introduce a "global" low-frequency coupling coefficient $\rho_{l f}$ such as: $\rho_{l f} K=\sum_{j} \rho_{j} K_{j}$ where $K$ is the total number of photoevents, i.e. $K=\sum_{j} K_{j}$. Supposing that $\forall_{i}, \bar{\rho}_{i}=\bar{\rho}$, we have $\bar{\rho}_{l f}=\bar{\rho}_{i}=\bar{\rho}$.

\section{B.2. Estimator of the visibility/Error on the modal visibility}

A classical estimator of the modal visibility consists in dividing the $H F$ spectral density of the interferogram by the photometric fluxes. We assume in the following that a fraction $\tau$ of the light has been selected for photometry analysis and that the rest of the light $(1-\tau)$ belongs to the interferogram. For sake of simplicity, we define $K^{\mathcal{P}}$ and $K^{I}$ as the total number of photoevents concerning respectively photometric and interferometric channels, i.e $K^{\mathcal{P}}=\tau K$ and $K^{I}=(1-\tau) K$. It comes:

$\widetilde{V_{i j}^{2}} \propto \frac{\left\langle\left|I^{2}\left(f_{i j}\right)\right|\right\rangle}{\left\langle k_{i}^{\mathcal{P}} k_{j}^{\mathcal{P}}\right\rangle}$.

Using Papoulis (1984) second order approximation, we can derive the square variance relative error of the modal visibility:

$$
\begin{aligned}
\frac{\sigma^{2}\left\{V_{i j}^{2}\right\}}{{\overline{V_{i j}^{2}}}^{2}}= & \frac{\sigma^{2}\left\{\left|I\left(f_{i j}\right)\right|^{2}\right\}}{\mathrm{E}^{2}\left\{\left|I\left(f_{i j}\right)\right|^{2}\right\}}+\frac{\sigma^{2}\left\{k_{i}^{\mathcal{P}} k_{j}^{\mathcal{P}}\right\}}{{\overline{k_{i}^{\mathcal{P}}}}^{2}{\overline{\mathcal{P}_{j}}}^{2}} \\
& -2 \frac{\operatorname{Cov}\left\{\left|I\left(f_{i j}\right)\right|^{2}, k_{i}^{\mathcal{P}} k_{j}^{\mathcal{P}}\right\}}{\mathrm{E}\left\{\left|I\left(f_{i j}\right)\right|^{2}\right\} \overline{k_{i}^{\mathcal{P}} k_{j}^{\mathcal{P}}}} .
\end{aligned}
$$

To derive those moments, we use the Goodman formalism described in Appendix A. In the case of fibered interferometers, the $L F$ and $H F$ of the Fourier-transformed interferogram $\left(H_{i}(f)\right.$ and $\left.H_{i j}(f)\right)$ are fixed by the geometry of the fibers, and statistics with respect to the turbulent atmosphere only appear in the coupling coefficients. For sake of simplicity we assume those coupling coefficients to be uncorrelated between two different baselines, although it does not change the eventual conclusions. Assuming also that the telescope transmissions are all equal, i.e. $K_{i}=K / N_{\text {tel }}$, it leads to the following expressions:

$\mathrm{E}\left\{\left|I\left(f_{i j}\right)\right|^{2}\right\}=\overline{\left|\rho_{i j}\right|^{2}} \frac{{\overline{K^{I}}}^{2}}{N_{\text {tel }}^{2}}+\bar{\rho} \overline{K^{I}}+N \sigma^{2}$

$$
\begin{aligned}
& \sigma^{2}\left\{\left|I\left(f_{i j}\right)\right|^{2}\right\}=\sigma_{\rho_{i j}^{2}}^{2} \frac{\overline{K^{I}}}{N_{\text {tel }}^{4}}+\left[2 \overline{\rho_{l f}^{2}}-\bar{\rho}^{2}\right]{\overline{K^{I}}}^{2} \\
& +\left[4 \overline{\rho_{l f}\left|\rho_{i j}\right|^{2}}-2 \bar{\rho} \overline{\left|\rho_{i j}\right|^{2}}\right] \frac{{\overline{K^{I}}}^{3}}{N_{\text {tel }}^{2}} \\
& +4 \overline{\left|\rho_{i j}\right|^{2}} \frac{{\overline{K^{I}}}^{2}}{N_{\text {tel }}^{2}}+2 N \sigma^{2} \overline{\left|\rho_{i j}\right|^{2}} \frac{{\overline{K^{I}}}^{2}}{N_{\text {tel }}^{2}} \\
& +2 N \sigma^{2} \bar{\rho} \overline{K^{I}}+3 N \sigma^{4}+N^{2} \sigma^{4} \\
& \sigma^{2}\left\{k_{i}^{\mathcal{P}} k_{j}^{\mathcal{P}}\right\}=\sigma_{\rho_{i} \rho_{j}}^{2} \frac{\overline{K^{\mathcal{P}}}}{N_{\text {tel }}^{4}}+2 \overline{\rho^{2}} \bar{\rho} \frac{{\overline{K^{\mathcal{P}}}}^{3}}{N_{\text {tel }}^{3}}+\bar{\rho}^{2} \frac{{\overline{K^{\mathcal{P}}}}^{2}}{N_{\text {tel }}^{2}}
\end{aligned}
$$

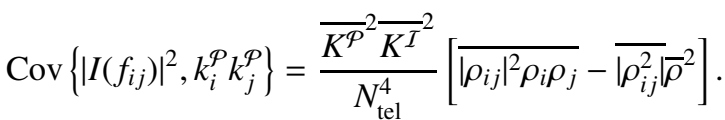

And the square relative error on the modal visibility can be expressed as the sum of three contributions:

$$
\frac{\sigma^{2}\left\{V_{i j}^{2}\right\}}{{\overline{V_{i j}^{2}}}^{2}}=\mathcal{E}_{\mathrm{P}}^{2}(K, \rho)+\mathcal{E}_{\mathrm{A}}^{2}\left(K, \sigma_{\mathcal{A}}^{2}, \rho\right)+\mathcal{E}_{\mathrm{S}}^{2}(\rho)
$$

where:

$$
\begin{aligned}
\mathcal{E}_{\mathrm{P}}^{2}= & {\left[\frac{N_{\text {tel }}\left(4 \overline{\rho_{l f}\left|\rho_{i j}\right|^{2}}-2 \bar{\rho} \mid \overline{\left.\rho_{i j}\right|^{2}}\right)}{(1-\tau){\overline{\left|\rho_{i j}\right|^{2}}}^{2}}+\frac{2}{\tau} \frac{\overline{\rho^{2}}}{\bar{\rho}^{3}}\right] \frac{N_{\text {tel }}}{\bar{K}} } \\
& +\left[\frac{N_{\text {tel }}^{2}\left(2 \overline{\rho_{l f}^{2}}-\bar{\rho}^{2}\right)}{(1-\tau)^{2} \overline{\left|\rho_{i j}\right|^{2}}}+\frac{4}{(1-\tau)^{2} \overline{\left|\rho_{i j}\right|^{2}}}+\frac{1}{\tau^{2} \bar{\rho}^{2}}\right] \frac{N_{\text {tel }}^{2}}{\bar{K}^{2}} \\
& +\frac{\bar{\rho}}{(1-\tau)^{3}{\overline{\left|\rho_{i j}\right|^{2}}}^{2}} \frac{N_{\text {tel }}^{4}}{\bar{K}^{3}}
\end{aligned}
$$

is the photon noise square relative error:

$$
\begin{aligned}
\mathcal{E}_{\mathrm{A}}^{2}= & \frac{3 N \sigma^{4}+N^{2} \sigma^{4}}{(1-\tau)^{4}{\overline{\left.\rho \rho_{i j}\right|^{2}}}^{2}} \frac{N_{\mathrm{tel}}^{4}}{\bar{K}^{4}}+\frac{2 N \sigma^{2}}{(1-\tau)^{2} \overline{\left|\rho_{i j}\right|^{2}}} \frac{N_{\mathrm{tel}}^{2}}{\bar{K}^{2}} \\
& +\frac{2 N \sigma^{2} \bar{\rho}}{(1-\tau)^{3} \overline{\left|\rho_{i j}\right|^{2}}} \frac{N_{\mathrm{tel}}^{4}}{\bar{K}^{3}}
\end{aligned}
$$

takes into account the additive noise $\sigma^{2}$ and

$\mathcal{E}_{\mathrm{S}}^{2}=\frac{\sigma_{\rho_{i j}^{2}}^{2}}{{\overline{\left|\rho_{i j}\right|^{2}}}^{2}}+\frac{\sigma_{\rho_{i} \rho_{j}}^{2}}{\bar{\rho}^{4}}-2 \frac{\operatorname{Cov}\left\{\rho_{i j}^{2}, \rho_{i} \rho_{j}\right\}}{\overline{\left|\rho_{i j}\right|^{2}} \bar{\rho}^{2}}$

arises from the coupling fluctuations inducing a so called "modal-speckle" square relative error. Concerning the latter contribution, it is interesting to notice that in the case of a point source, we have $\left|\rho_{i j}\right|^{2}=\rho_{i} \rho_{j}$. The outcome is that the "modalspeckle" noise contribution for a point source is zero.

\section{Appendix C: Coupling coefficients statistics}

In order to calculate the square relative error of the modal visibility given in Appendix B, we first compute first and second 
Table C.1. $L F$ and $H F$ coupling coefficient first and second order statistics. Rigorous expressions.

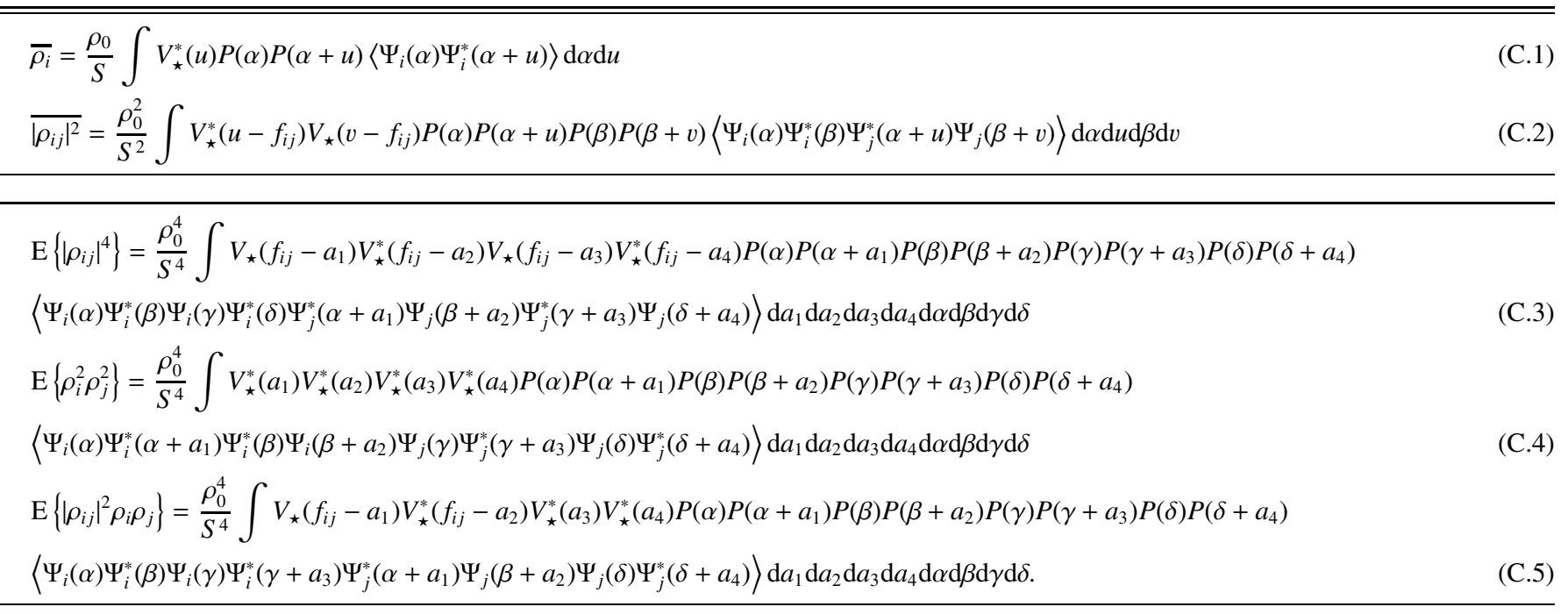

order moments of the $L F$ and $H F$ coupling coefficients which are defined as following:

$$
\begin{aligned}
\rho_{i}\left(V_{\star}\right) & =\rho_{0} \frac{\left(V_{\star} * T^{i}\right)_{f=0}}{\int T_{0}^{i}(f) \mathrm{d} f} \\
\rho_{i j}\left(V_{\star}\right) & =\rho_{0} \frac{\left(V_{\star} * T^{i j}\right)_{f=f_{i j}}}{\sqrt{\int T_{0}^{i}(f) \mathrm{d} f \int T_{0}^{j}(f) \mathrm{d} f}}
\end{aligned}
$$

where $T^{i}$ and $T^{i j}$ are the (partially AO corrected) modal transfer function resulting respectively from the normalized autocorrelation and cross-correlation of the entrance aberrationcorrupted pupil weighted by the fiber single mode, i.e.:

$$
\begin{aligned}
T^{i}(u) & =\frac{1}{S} \int P_{i}(\alpha) P_{i}(\alpha+u) \Psi_{i}(\alpha) \Psi_{i}^{*}(\alpha+u) \mathrm{d} \alpha \\
T^{i j}(u) & =\frac{1}{S} \int P_{i}(\alpha) P_{j}(\alpha+u) \Psi_{i}(\alpha) \Psi_{j}^{*}(\alpha+u) \mathrm{d} \alpha .
\end{aligned}
$$

Introducing Eqs. (C.8), (C.9) in Eqs. (C.6) and (C.7) and developing the expressions of first order $\left(\overline{\rho_{i}}, \overline{\left|\rho_{i j}\right|^{2}}\right)$, and second order $\left(\sigma_{\left|\rho_{i j}\right|^{2}}^{2}, \sigma_{\rho_{i} \rho_{j}}^{2}, \operatorname{Cov}\left\{\left|\rho_{i j}\right|^{2}, \rho_{i} \rho_{j}\right\}\right)$ coupling coefficient statistics, leads respectively to second, fourth and eighth order moments of the complex amplitude of the wavefronts $\Psi_{i}(u)$ and $\Psi_{j}(u)$. Such rigorous expressions are written in Table C.1.

At this point, those expressions are not yet formally computable. Hence we perform simplifications of the equations. We first assume that the wavefronts are uncorrelated, i.e. $\left\langle\Psi_{i}(u) \Psi_{j}(u)\right\rangle_{\phi}=\left\langle\Psi_{i}(u)\right\rangle_{\phi}\left\langle\Psi_{j}(u)\right\rangle_{\phi}$. Then we use Korff's (1973) derivation of the moments of the complex amplitude of the wavefronts to introduce in the equations, linear combinations of the structure function $(\mathcal{D}(u, v))$ at different spatial frequencies. We recall that the structure function is defined such that (Conan 1994):

$\mathcal{D}(u, v)=\left\langle\psi_{i}(u) \psi_{i}^{*}(v)\right\rangle_{\phi}=\left\langle\psi_{j}(u) \psi_{j}^{*}(v)\right\rangle_{\phi}$.

Finally we suppose that these structure functions are stationary, i.e. $\mathcal{D}(u, v)=\mathcal{D}(u-v)$. After such formal derivations we obtain expressions of the coupling coefficients statistics as summarized in Table C.2. Readers may note that in the pure turbulent case, we assume in addition that the complex amplitude of the wavefront follows circular Gaussian statistics (Roddier 1988), hence slightly changing the expressions given in Table C. 2

\section{Appendix D: Estimator of the visibility for multispeckle interferometry}

\section{D.1. The interferometric equation}

For non fibered interferometers, the convolution between the object and the interferometer transfer function stands for any spatial frequency (Tallon \& Tallon-Bosc 1992). We may express:

$I(f)=\sum_{i} K_{i} i(f)+\sum_{i} \sum_{j} \sqrt{K_{i} K_{j}} i\left(f-f_{i j}\right)$

with

$i(f)=V(f) . S(f)$

where $V(f)$ is the object visibility and $S(f)$ is the normalized transfer function of the interferometer which consists in the autocorrelation of the pupil function weighted by the remaining atmospheric phase. We have the following relationship

$S(f)=\sum_{i} S^{i}(f)+\frac{1}{N_{\text {tel }}} \sum_{i} \sum_{j} S^{i j}(f)$

where $S^{i i}(f)$ and $S^{i j}(f)$ are respectively the auto and cross-correlation of single phase corrugated pupil (Roddier 1988), i.e.:

$S^{i i}(f)=\frac{1}{S} \int P(\alpha) P(\alpha+f) \Psi_{i}(\alpha) \Psi_{i}^{*}(\alpha+f) \mathrm{d} \alpha$

$S^{i j}(f)=\frac{1}{S} \int P(\alpha) P(\alpha+f) \Psi_{i}(\alpha) \Psi_{j}^{*}(\alpha+f) \mathrm{d} \alpha$. 
Table C.2. $L F$ and $H F$ coupling coefficient first and second order statistics. simplified expressions after assuming: (i) no correlation between wavefronts; (ii) Korff's derivation of the moments of the complex amplitude of the wavefronts; (iii) stationarity of the structure function.

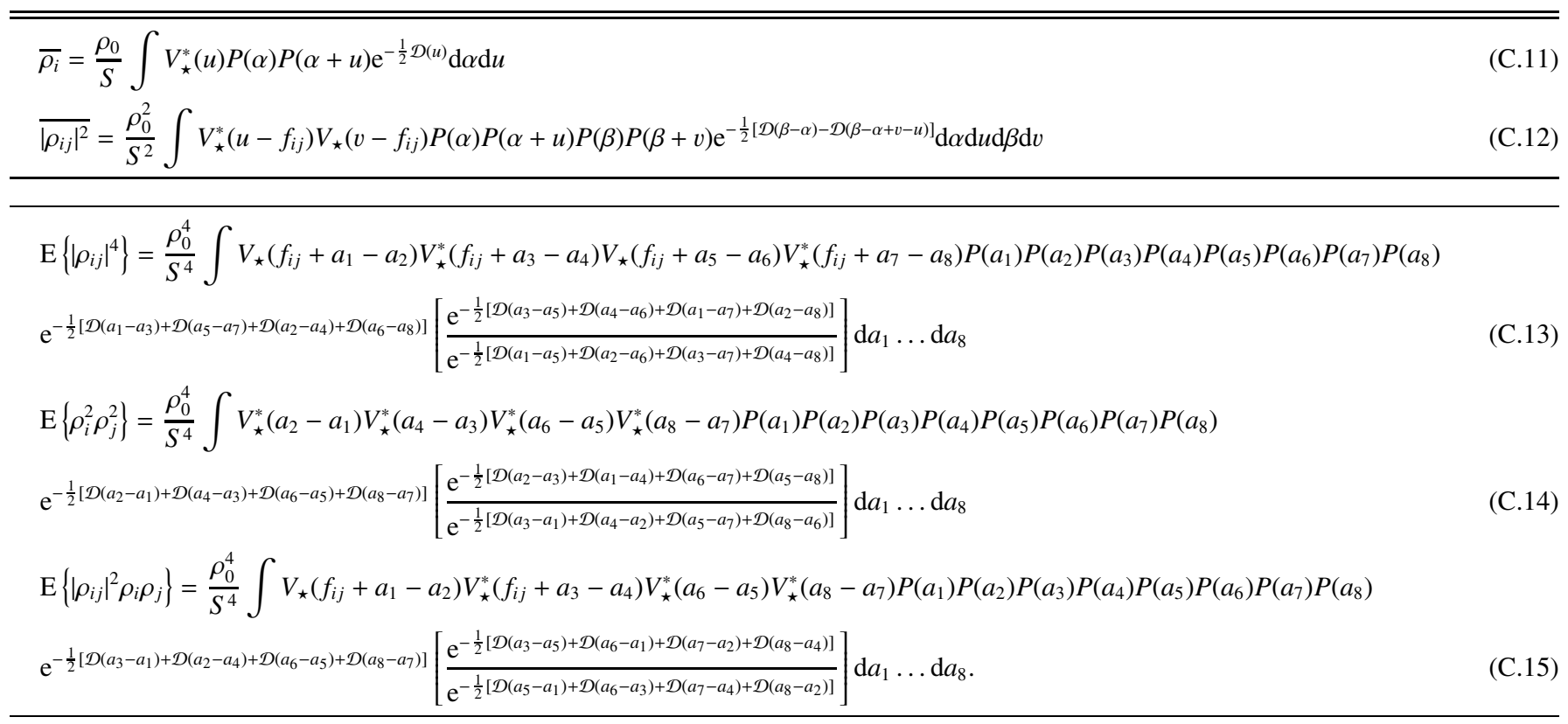

\section{D.2. Estimator of the visibility/Error on the visibility}

To perform a consistent comparison within the fiber case, we define the estimator of the multispeckle visibility as the ratio between the integrated high frequency peak and the photometric fluxes. We still assume that a fraction $\tau$ of the light is injected in the photometric channels. It writes:

$\widetilde{V^{2}}\left(f_{i j}\right) \propto \frac{\int_{f_{i j}-\frac{D}{\lambda}}^{f_{i j}+\frac{D}{\lambda}}|I(f)|^{2} \mathrm{~d} f}{\left\langle k_{i}^{\mathcal{P}} k_{j}^{\mathcal{P}}\right\rangle}$.

From a digital point of view, as the size of the instantaneous speckle image is $\frac{\lambda}{r_{0}}$ wide (perfect correction apart), the integral of the previous equation can be replaced by a discrete sum over points of the peak that are distributed every $\frac{r_{0}}{\lambda}$. We then have an equivalent estimator:

$\widetilde{V^{2}}\left(f_{i j}\right) \propto \frac{\sum_{l=f_{i j}-\frac{D}{\lambda}}^{f_{i j}+\frac{D}{\lambda}}|I(l)|^{2}}{\left\langle k_{i}^{\mathcal{P}} k_{j}^{\mathcal{P}}\right\rangle}, \Delta l=\frac{r_{0}}{\lambda}$.

We derive the square relative error from Papoulis (1984):

$$
\begin{aligned}
\frac{\sigma^{2}\left\{V_{i j}^{2}\right\}}{{\overline{V_{i j}^{2}}}^{2}}=\frac{\sum_{l} \sigma^{2}\left\{|I(l)|^{2}\right\}+\sum_{m, n \neq m} \operatorname{Cov}\left\{|I(m)|^{2}|I(n)|^{2}\right\}}{\left[\sum_{l} \mathrm{E}\left\{|I(l)|^{2}\right\}\right]^{2}} \\
+\frac{1}{k_{i}^{\mathcal{P}}}+\frac{1}{k_{j}^{\mathcal{P}}}+\frac{1}{k_{i}^{\mathcal{P}} k_{j}^{\mathcal{P}}} \cdot
\end{aligned}
$$

Note that correlations between points have to be taken into account since we consider partial correction by Adaptive Optics.
We then use the formalism developed in Appendix A to derive (D.8). Note that in the multispeckle case, at the contrary of the fibered case, the $L F$ and $H F$ peaks of the Fouriertransformed interferogram (i.e. $S^{i}(f)$ and $S^{i j}(f)$ ) depend on the turbulence. Moments of $|I(l)|^{2}$ are:

$$
\begin{aligned}
\mathrm{E}\left\{|I(l)|^{2}\right\}= & \frac{\bar{K}^{I}}{N_{\text {tel }}^{2}}\left\langle\left|S^{i j}(l)\right|^{2}\right\rangle V_{l}^{2}+\overline{K^{I}}+N \sigma^{2} \\
\sigma^{2}\left\{|I(l)|^{2}\right\}= & \overline{K^{I}}+{\overline{K^{I}}}^{2} \\
& +\left[2 \frac{\bar{K}^{I}}{N_{\text {tel }}^{2}}+4 \frac{{\overline{K^{I}}}^{2}}{N_{\text {tel }}^{2}}\right]\left\langle\left|S^{i j}(l)\right|^{2}\right\rangle_{\phi} V_{l}^{2} \\
& +\frac{\bar{K}^{I}}{N_{\text {tel }}^{4}}\left[\left\langle\left|S^{i j}(l)\right|^{4}\right\rangle_{\phi}-\left\langle\left|S^{i j}(l)\right|^{2}\right\rangle_{\phi}^{2}\right] V_{l}^{4} \\
& +2 N \sigma^{2} \frac{\bar{K}^{I}}{N_{\text {tel }}^{2}}\left\langle\left|S^{i j}(l)\right|^{2}\right\rangle_{\phi} V_{l}^{2}+2 N \sigma^{2} \overline{K^{I}} \\
& +3 N \sigma^{4}+N^{2} \sigma^{4}
\end{aligned}
$$

$$
\begin{aligned}
\operatorname{Cov}\left\{|I(m)|^{2}|I(n)|^{2}\right\}=\overline{K^{I}}+\frac{{\overline{K^{I}}}^{2}}{N_{\text {tel }}^{2}}\left[2\left\langle\left|S^{i j}(m)\right|^{2}\right\rangle_{\phi} V_{m}^{2}\right. \\
\left.+2\left\langle\left|S^{i j}(n)\right|^{2}\right\rangle_{\phi} V_{n}^{2}+\left\langle\left|S^{i}(m-n)\right|^{2}\right\rangle_{\phi} V_{m-n}^{2}\right] \\
+2 \frac{\bar{K}^{I}}{N_{\text {tel }}^{2}}\left\langle S^{i j}(m) S^{i j}(n) S^{i^{*}}(m-n)\right\rangle V_{m} V_{n} V_{m-n}^{*} \\
+\frac{K^{I}}{N_{\text {tel }}^{4}}\left[\left\langle\left|S^{i j}(m)\right|^{2}\left|S^{i j}(n)\right|^{2}\right\rangle_{\phi}\right. \\
\left.-\left\langle\left|S^{i j}(m)\right|^{2}\right\rangle_{\phi}\left\langle\left|S^{i j}(n)\right|^{2}\right\rangle_{\phi}\right] V_{m}^{2} V_{n}^{2}+3 N \sigma^{4} .
\end{aligned}
$$


Table E.1. Formal expressions of respectively $L F$ and $H F$ speckle transfer function first and second order statistics.

\begin{tabular}{l}
\hline$\left\langle\left|S^{i}(f)\right|^{2}\right\rangle=S^{-2} \int P\left(a_{1}\right) P\left(a_{1}+a_{2}\right) P\left(a_{1}+f\right) P\left(a_{1}+a_{2}+f\right)\left[\frac{\mathrm{e}^{-\left[\mathcal{D}\left(a_{2}\right)+\mathcal{D}(f)\right]}}{\left.\mathrm{e}^{-\frac{1}{2}\left[\mathcal{D}\left(a_{2}+f\right)+\mathcal{D}\left(a_{2}-f\right)\right]}\right] \mathrm{d} a_{1} \mathrm{~d} a_{2}}\right.$ \\
$\left\langle\overline{\left\langle\left. S^{i j}(f)\right|^{2}\right\rangle=S^{-2} \int P\left(a_{1}\right) P\left(a_{2}\right) P\left(a_{1}+f\right) P\left(a_{2}+f\right) \mathrm{e}^{-\mathcal{D}\left(a_{1}-a_{2}\right)} \mathrm{d} a_{1} \mathrm{~d} a_{2}}\right.$ \\
\hline$\left\langle\left. S^{i j}(f)\right|^{4}\right\rangle=S^{-4} \int P\left(a_{1}\right) P\left(a_{1}+f\right) P\left(a_{2}\right) P\left(a_{2}+f\right) P\left(a_{1}+a_{3}\right) P\left(a_{1}+a_{3}+f\right) P\left(a_{2}+a_{4}\right) P\left(a_{2}+a_{4}+f\right)$ \\
{$\left[\frac{\mathrm{e}^{-\left[\mathcal{D}\left(a_{1}-a_{2}\right)+\mathcal{D}\left(a_{3}+a_{1}-a_{2}\right)+\mathcal{D}\left(a_{2}+a_{4}-a_{1}\right)+\mathcal{D}\left(a_{4}+a_{2}-a_{1}-a_{3}\right)\right]}}{\mathrm{e}^{-\left[\mathcal{D}\left(a_{3}\right)+\mathcal{D}\left(a_{4}\right)\right]}}\right] \mathrm{d} a_{1} \mathrm{~d} a_{2} \mathrm{~d} a_{3} \mathrm{~d} a_{4}$}
\end{tabular}

Another classical estimator of the visibility (Roddier \& Lena 1984; Mourard et al. 1994), known to be more robust to the turbulence, consists in taking the ratio of the high-frequency peak energy by the low-frequency one (coherent energy versus incoherent one):

$\widetilde{V^{2}}\left(f_{i j}\right)=\frac{\int_{f_{i j}-\frac{D}{\lambda}}^{f_{i j}+\frac{D}{\lambda}}|I(f)|^{2} \mathrm{~d} f}{\int_{-\frac{D}{\lambda}}^{\frac{D}{\lambda}}|I(f)|^{2} \mathrm{~d} f}$.

Assuming that photometric channels contain at most $50 \%$ of the information, we expect the relative error of the visibility from this estimator to be equal to the previous one within a factor of $\sqrt{2}$.

\section{Appendix E: Speckle transfer function statistics}

In order to calculate the square relative error of the visibility, we first need to compute expected values of $\left|S^{i}(f)\right|^{2}$, $\left|S^{i j}(f)\right|^{2}$ and $\left|S^{i j}(f)\right|^{4}$. As discussed in Appendix D the functions $S^{i}(f)$ and $S^{i}(f)$ are respectively the auto and the crosscorrelation of the pupil functions weighted by the remaining atmospheric wavefront. If we neglect the weighting of the pupil by the single mode of the fiber, we find the very same expression as in Eqs. (C.8), (C.9), i.e. $S^{i}(u)=T^{i}(u)$ and $S^{i j}(u)=$ $T^{i j}(u)$. Deriving $\left\langle\left|S^{i}(f)\right|^{2}\right\rangle,\left\langle\left|S^{i j}(f)\right|^{2}\right\rangle,\left\langle\left|S^{i}(f)\right|^{4}\right\rangle,\left\langle\left|S^{i j}(f)\right|^{4}\right\rangle$ introduces once again second, fourth and eighth order statistics of the complex amplitude of the wavefronts, that we develop following the same procedure as described in Appendix C. We finally obtained expressions given in Table E.1. 